\title{
Pollen morphology in two Urgineoid species of Drimia (Baker) Jessop
}

\author{
P. Prathima ${ }^{1}$, M. N. Shiva Kameshwari ${ }^{2 *}$ and R. Nijagunaiah ${ }^{3}$ \\ ${ }^{1}$ Research and Development Centre, Bharathiar University, Coimbatore - 641 046, INDIA \\ $2 \& 3 *$ Department of Botany, Jnana Bharathi Campus, Bangalore University, 560056. INDIA \\ *Corresponding author: E-mail.mn.shivakameshwari@gmail.com
}

Received: May 15, 2015; Revised received: October 30, 2015; Accepted: February 4, 2016

\begin{abstract}
Pollen grains of eight accessions of Urginea indica (Roxb) Kunth. and two accessions of Urgineawightii (Wight) Lakshmin. were examined by Light microscopy and Scanning electron microscopy. Detailed pollen morphological characters are given for these accessions. Our investigation revealed several variations in pollen size, exine ornamentation among the accessions of $U$. indica (Roxb) Kunth. and $U$. wightii Wight) Lakshmin. The study confirmed that the exine sculpting provides valuable characters for separating the species of Urginea. Reticulate ornamentation of the exine was observed in the accessions of $U$. indica complex and perforated exine ornamentation in accessions of $U$. wightii complex. Minor variations with regard to measurements of dimensions in size, shape and ornamentation were also observed in accessions of $U$. indica and $U$. wightii . Pollen fertility analysis revealed highest percentage fertility in Sitampoondi accession of $U$. indica complex and Gulbargha accession of $U$. wightii complex but in most of the accessions seed setting is not observed in natural conditions. Pollen germination studies revealed highest germination rate in Brew Baker's and Kwack medium supplemented with 25\% sucrose concentration for Urginea pollen.
\end{abstract}

Keywords: Accessions, Hyacinthaceae, Pollen fertility, Scanning electron microscope, Urginea indica, Urginea wightii

\section{INTRODUCTION}

Urginea Steinhill.Hyacinthaceae is one of the interesting polytypic genus with about 100 species occurring in India, Africa, Mediterranean regions and in the plains of South India (Airy Shaw, 1965). The genus Urginea is a bulbous geophyte with species showing phenotypic plasticity. The genus was for the first time investigated cytologically by Raghavan (1935). Shiva Kameshwari and Muniyamma (2004) have made extensive studies and have demonstrated the presence of natural polyploidy in the genus. Urginea is known for a wide range of morphological and cytological variations. This must have misled the previous workers to make erroneous identifications and arrive at wrong conclusions. It is well known that pollen features have a great taxonomic value, and have been used in the classification of different genera (Troia et al.,2012; Ceter et al.2013) and also closely related Liliaceae taxa (Teksen et al. 2010;Masoumi 2012). A comparative study of the pollen morphology employing electron microscopy has been made by Kosenko (1999). The pollen morphology of Nomocharis with Lilium of Liliaceae were investigated under both LM and SEM to elucidate the systematics of the genera by Liang and Zhang (1985). Similar studies were carried out in Lilium (Liliaceae) by Seher Guven et al. (2014). Palynological studies investigated in Liliaceae members indicated that palynological characters such as sulcus, muri and lumina are taxonomically useful
Kuprianova (1983); Kosenko (1999); Shiva Kameshwari (2011). Pollen grains although minute show many characters of taxonomic and phylogenetic importance. The present study aims at detailed pollen morphological characters for eight accessions of $U$. indica (Roxb) Kunth. and two accessions of $U$. wightii (Wight) Lakshmin. The investigation also evaluates the taxonomical relationship between the species and accessions of Urginea from a pollen morphological perspective and test the viability of pollen grains and in vitro pollen germination of few accessions.

\section{MATERIALS AND METHODS}

In the present investigation eight accessions of $U$. indica and two accessions of $U$. wightii were collected, identified, and maintained in the germplasm at Department of Botany, Bangalore University, Bangalore. Phenology: Phenological events (time of leaf fall, renewal, flowering and fruiting period) were recorded. Morphometrical studies of flowering phenology, counting the number of flowers, length of the inflorescence, perianth, androecium and gynoecium of the accessions periodically for about 4 years throughout the flowering period (March to May), blooming time of the flowers were observed and recorded.

Light microscopic studies: Pollen of eight accessions of $U$. indica and two accessions of $U$. wightii were collected from various localities and were used for LM studies. For LM investigations pollen slides were 
prepared by mounting pollen grains from freshly dehisced anther suspended in 50\% glycerin and mounted on a slide. Pollen size was measured with an ocular micrometer under light microscope as described by Moore and Webb (1978).

Scanning electron microscopic studies: Pollen grains were transferred to aluminum stubs and coated with gold and examined under a JOEL JSM 5600 Scanning electron microscope. Pollen morphometric measurements were made using Image $J$ software. The figures reported are the average of 20 measurements of the pollen grains per accessions

Pollen fertility: Fresh pollen of 10 accessions of $U$. indica and 3 accessions of $U$. wightii were collected at anthesis. TTC (2,3,5-triphenyl tetrazolium chloride) stain test was used for this purpose after the method of Hauser and Morrison (1964). The pollen fertility tests were tested individually for each accession for both the species. A few drops of $0.5 \%$ TTC were dropped by pipettes on microscope slides and pollen were dusted with a slim brush (each brush used only one accession type) covered with a cover slip and 3 different areas of each cover slip were counted for TTC tests. The fertile pollen grains stained with TTC dyed majenta color were considered as viable. Those pollen grains which do not show this effect were classified as unviable or sterile. Pollen fertility was determined as per the formula.

Percentage Pollen Fertility $=$ Number of Fertile pollen grain stained / Total number of Pollen grains X 100

In vitro pollen germination: Brew Baker's and Kwack (1963) culture medium with different sucrose concentrations $(5 \%, 15 \%$ and $25 \%)$ were used for the pollen germination studies in accession number 835 (Ramanagaram) of U.indica complex.

\section{RESULTS AND DISCUSSION}

Pollen morphological attributes can be used both to distinguish related taxa or to integrate them into a common group (Sevil Pehlivan 2003). Pollen exine sculpturing is considered to be as evolutionary reversible character but the internal wall structures are thought to be more stable and are important in assessing affinities
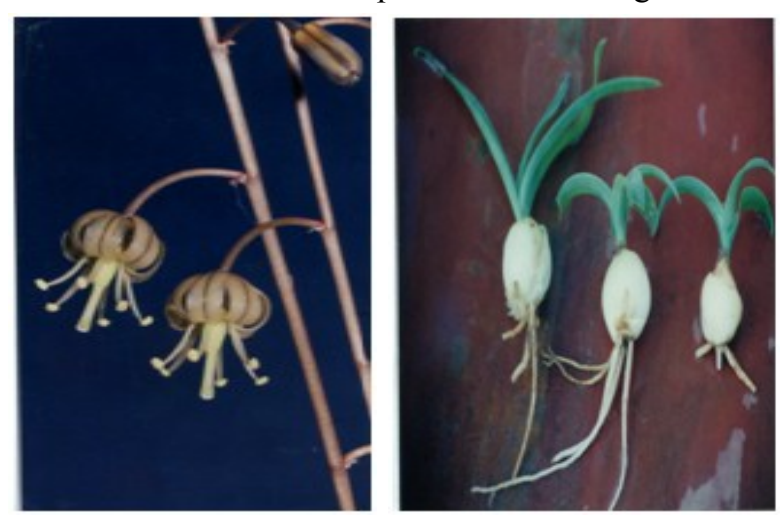

Urginea indica (Roxb) Kunth. Accession Number (835) with other families in the order of Liliales (Walker and Doyle 1975).

Monosulcate pollen grains are a primitive trait in seed plants and occur widely among the monocotyledons (Furness and Rudall 2001). According to LM and SEM investigations, the pollen grains of various accessions confirmed that all accessions of the genus $U$. indica and $U$. wightii were found to be monosulcate and ellipsoidal (Figs. 1 and 2). The color of the pollen grains of the examined accessions belonging to both the species of Urginea were yellow .The predominance of monosulcate aperture is emphasized by Harley and Zavada (2000). Similar results were reported in genus Lilium by Kosenko (1999), Pupuleku et al. (2010) and Muratovic et al. (2010).

The two Urginea species were homogenous in possessing a single longitudinal aperture (monosulcate). Finer differences and variations do exist in the reticulation of the exine between the species and accessions as observed in $U$. indica complex and $U$. wightii complex. Variation in the aperture examined in the pollen grains of accessions in both the species can be used to delimit the species. The main features of the investigated pollen are summarized in Tables $1-4$. Large boat shaped, ellipsoidal monosulcate pollen grains with reticulate and perforated exine ornamentation are the most common type of pollen found in $U$. indica and U. wightii. Palynological measurements are summarized in Table 1 and Table 2. During the present study remarkable degrees of variations in the measurements of dimensions were observed between the accessions of Urginea. Among the examined accessions of $U$. indica complex, biggest pollen size was found in accession number 842 (Kerala) and smallest in accession number 845 (Coimbatore). In accessions of $U$. wightii complex biggest pollen size was observed in accession number 825 (Gulbarga).

Studies in pollen viability and morphology play a role in genetic breeding programmes. Determination of pollen fertility or viability can be assessed by the use of direct methods such as the inducement of in vitro germination (Acar and Kakani 2010; Alcaraz et al., 2011; Sorkheh et al. 2011) and in vivo germination
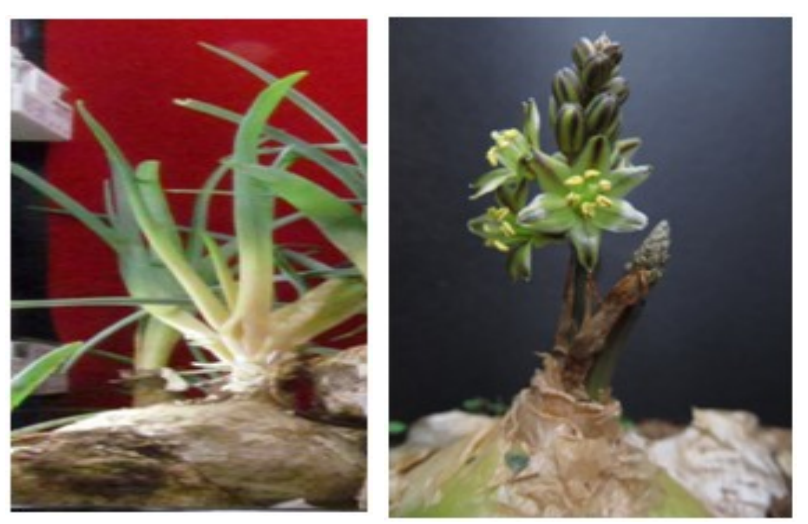

Urginea wightii (Wight) Lakshmin. Accession Number (839) 
Table 1. Pollen morphological parameters in accessions of $U$. indica (Roxb.)Kunth

\begin{tabular}{llllllll}
\hline $\begin{array}{l}\text { SI. } \\
\text { no }\end{array}$ & $\begin{array}{l}\text { Name of the } \\
\text { Accession and number }\end{array}$ & $\begin{array}{l}\text { Long axis } \\
(\boldsymbol{\mu m})\end{array}$ & $\begin{array}{l}\text { Short axis } \\
(\boldsymbol{\mu m})\end{array}$ & Ornamentation & $\begin{array}{l}\text { Flowering } \\
\text { Time }\end{array}$ & $\begin{array}{l}\text { Chromosome } \\
\text { number }\end{array}$ & Ploidy \\
& & & & & & \\
\hline 1 & Kerala (842) & 84.99 & 40.22 & Reticulate & Evening & 50 & Pentaploid \\
2 & Ramanagaram (835) & 74.49 & 28.80 & Reticulate & Evening & 20 & Diploid \\
3 & Trichy (844) & 77.96 & 31.55 & Reticulate & Evening & - & - \\
4 & Biligirirangana hills (836) & 84.28 & 33.14 & Reticulate & Evening & 36 & Aneuploid \\
5 & Gopalaswamy betta (807) & 77.35 & 29.96 & Reticulate & Afternoon & 40 & Tetraploid \\
6 & Coimbatore (845) & 73.10 & 31.47 & Reticulate & Evening & - & - \\
7 & Ranganthittu (804) & 83.56 & 26.13 & Reticulate & Night & 34 & Aneuploid \\
8 & Shimoga (802) & 78.23 & 32.79 & Reticulate & Morning & 20 & Diploid \\
\hline
\end{tabular}

Table 2. Pollen morphological parameters in accessions of $U$. wightii (Wight) Lakshmin.

\begin{tabular}{|c|c|c|c|c|c|c|c|}
\hline $\begin{array}{l}\text { S. } \\
\text { N. }\end{array}$ & $\begin{array}{l}\text { Name of the } \\
\text { Accession and number }\end{array}$ & $\begin{array}{l}\text { Long axis } \\
(\mu \mathrm{m})\end{array}$ & $\begin{array}{l}\text { Short axis } \\
(\mu \mathrm{m})\end{array}$ & Ornamentation & $\begin{array}{l}\text { Flowering } \\
\text { time }\end{array}$ & $\begin{array}{l}\text { Chromosome } \\
\text { number }\end{array}$ & Ploidy \\
\hline 1 & Gubbi (848) & 62.50 & 24.02 & Perforated & Afternoon & 20 and 40 & $\begin{array}{l}\text { Diploid and } \\
\text { Tetraploid }\end{array}$ \\
\hline 2 & Gulbargha (825) & 78.75 & 31.67 & Perforated & Morning & 36 & Aneuploid \\
\hline S.N. & \multicolumn{3}{|c|}{ Name of the Accession and number } & o Fertility & \multicolumn{2}{|c|}{ Chromosome number } & Ploidy \\
\hline 1 & \multicolumn{2}{|l|}{ Banganwadi (815) } & & $25.92 \%$ & \multicolumn{2}{|l|}{40} & Tetraploid \\
\hline 2 & \multicolumn{2}{|l|}{ Karighatta (826) } & & $77.21 \%$ & \multicolumn{2}{|l|}{34} & Aneuploid \\
\hline 3 & \multicolumn{2}{|c|}{ Sitampoondi (846) } & & $95.5 \%$ & \multicolumn{2}{|l|}{20} & Diploid \\
\hline 4 & \multicolumn{2}{|c|}{ Channamallipura (809) } & \multicolumn{2}{|r|}{$6.52 \%$} & \multirow{4}{*}{40} & & \\
\hline 5 & \multicolumn{2}{|c|}{ Coimbatore (845) } & & $93.22 \%$ & & & \multirow[b]{2}{*}{ Tetraploid } \\
\hline 6 & \multicolumn{2}{|c|}{ Gopalaswamybetta (807) } & & $76.19 \%$ & & & \\
\hline 7 & \multicolumn{2}{|l|}{ Ramanagaram (835) } & & $6.45 \%$ & & & \\
\hline 8 & \multicolumn{2}{|l|}{ Ranganthittu 804} & & $7.936 \%$ & \multicolumn{2}{|l|}{34} & Aneuploid \\
\hline 9 & Magadi (834) & & & $91.66 \%$ & \multicolumn{2}{|l|}{20} & Diploid \\
\hline 10 & \multicolumn{2}{|l|}{ Shimoga (802) } & & $5.376 \%$ & \multicolumn{2}{|l|}{20} & Diploid \\
\hline
\end{tabular}

(Fakhim et al. 2011) or other, indirect methods based on cytological parameters, such as pollen staining (Beyhan and Serdar 2008; Abdelgadir et al. 2012). Pollen fertility was assessed through histochemical analysis using $\quad 0.5 \%$. 2,3,5-triphenyltetrazolium chloride (TTC) solution. Pollen Fertility analysis indicated highest in accession number 846 (Sitampoondi) and lowest in accession number 802 (Shimoga) of $U$. indica complex and highest in accession number 825 (Gulbarga) and lowest in accession number 848 (Gubbi) of $U$. wightii complex (Table 3 and 4). Seed setting was observed in these three accessions which indicated highest fertility rate (accession number 846, 825 and 839) but seed setting is not observed in majority of the accessions in natural conditions.

In vitro analysis of pollen germination studied in accession number 835 (Ramanagaram) presented the highest germination rate in Brew Baker's and Kwack (1963) medium with 25\% sucrose concentration (Fig 3). During pollen development, T.S. of microsporangium revealed normal and shrunken pollen grains (Fig. 4) with vegetative and generative nucleus. The exine structure of pollen grains comprised tectum and collumellae. Variations in exine thickness is an important palynological trait examined in different accessions of $U$. indica and $U$. wightii. Ornamentation characteristics of the pollen grains of the investigated accessions as observed in SEM micrographs have been used for their diagnostic features and accordingly reticulate ornamentation were observed in $U$. indica and perforated ornamentation in $U$. wightii. According to Neetin Desai et.al (2012) Indian Drimia (Urginea) species cannot be distinguished on the basis of their pollen morphology as they have more similarity than differences. In the present investigation reticulate ornamentation was observed in $U$. indica complex and perforated ornamentation in $U$. wightii complex.

For resolving taxonomic misunderstandings morphological characters have the most important role in plant systematics, palynological,and anatomical characters can be used to support the morphological characters. Ploidy plays an important role in delimiting the taxa. Genome size is the predictor of pollen size. According to Charles Knight et al., (2010), pollen is not a good candidate to relate with the genome size but 
Table 4. Percentage fertility in Urginea wightii accessions

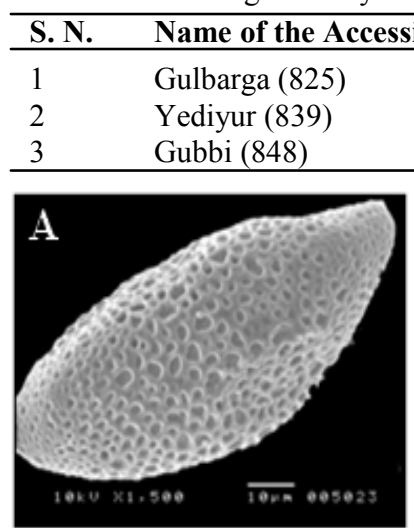

Kerala

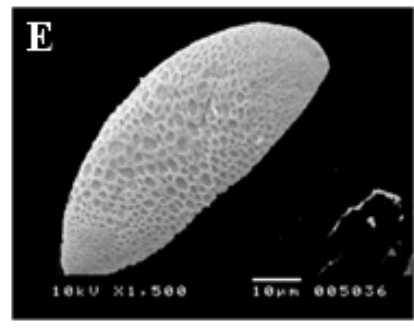

Gopalaswamy betta

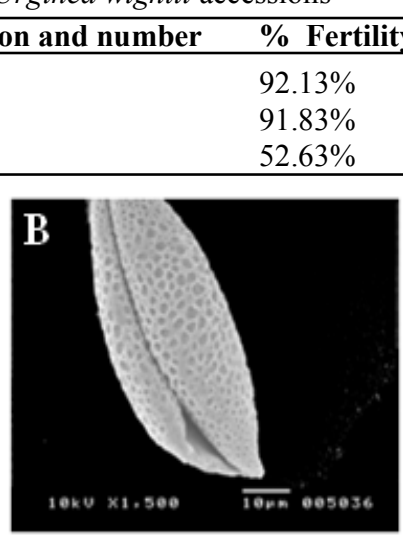

Ramanagaram

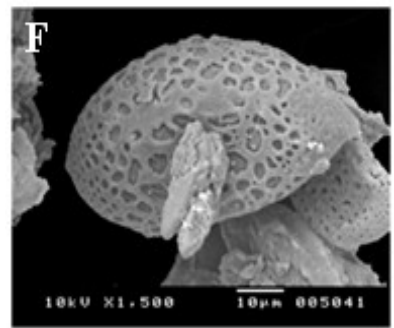

Coimbatore

\begin{tabular}{ll} 
Chromosome number & Ploidy \\
36 & Aneuploid \\
20 & Diploid \\
20 and 40 & Diploid and Tetraploid \\
\hline
\end{tabular}

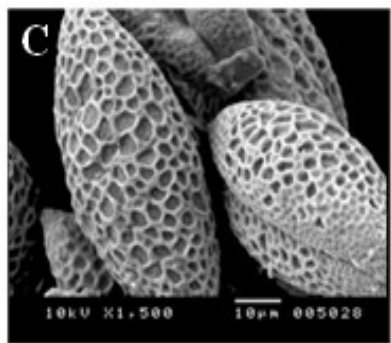

Trichy

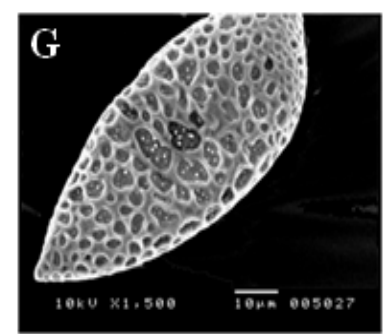

Ranganthittu

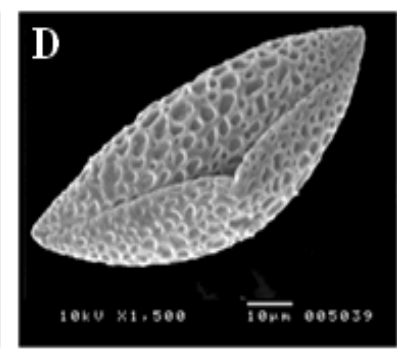

Biligirirangana Hills

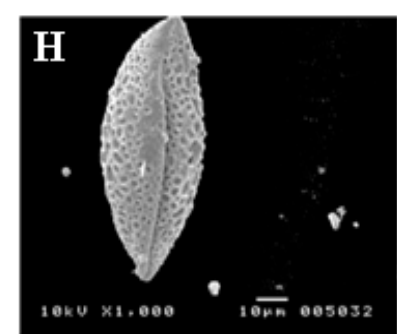

Shimoga

Fig 1: $\boldsymbol{A}-\boldsymbol{H}$. SEM photographs of pollen grains of Urginea indica accessions showing reticulate sculpturing. A - Proximal view of accession number 842 (Kerala). B - Distal view of accession number 835 (Ramanagaram). $\boldsymbol{C}$ - Proximal view of accession number 844 (Trichy). D - Distal view of accession number 836 (Biligirirangana hills). $\boldsymbol{E}$ - Proximal view of accession number 807 (Gopalaswamy betta). $\boldsymbol{F}$ - Proximal view of accession number 845 (Coimbatore). $\boldsymbol{G}$-Proximal view of accession number 804 (Ranganthittu). $\boldsymbol{H}$-Distal view of accession number 802 (Shimoga).

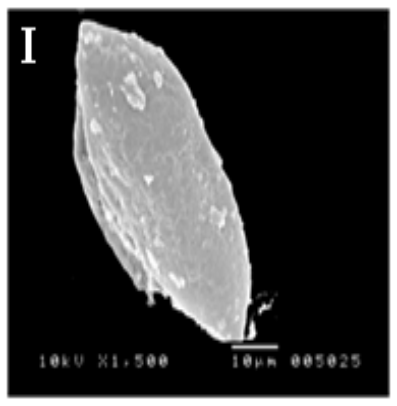

Gubbi

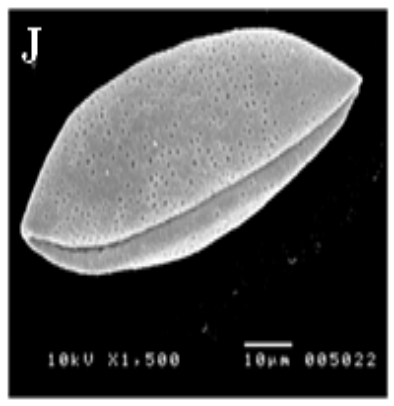

Gulbarga

Fig 2: I - J. SEM photographs of pollen grains of Urginea wightii accessions showing perforated ornamentation. I Proximal view of accession number 848 (Gubbi). J-Distal view of accession number 825 (Gulbargha).

in our present investigation, there is considerable variation in pollen size with respect to ploidy. accession number 842 being pentaploid showing biggest pollen grain in the $U$. indica complex whereas in $U$. wightii complex, accession number 825 an aneuploid shows biggest pollen grains indicating that ploidy plays an important role in delimiting the taxa.

\section{Conclusion}

Our investigation confirms that pollen characters in Urginea were of taxonomic significance. Differences

were encountered in pollen shape, size, ornamentation, sulcus membrane ornamentation, apex of sulcus, muri and lumina. The exine ornamentation observed in $U$. indica complex and $U$. wightii complex reveals that it is one of the valuable characters for separating the two species. Reticulate ornamentataion was observed in $U$ indica complex and perforated ornamentation in $U$. wightii complex. There is also intraspecific variation observed between the accessions of both the complexes which indicates the heterogeneity of the species. The study of the pollens of Urginea highlights that features like size related to ploidy will be useful in assessing and evaluating the taxonomic relationships between the species and the accessions. Pollen morphological features provide additional valuable taxonomic information in separating the accessions of $U$. indica (Roxb)Kunth. and $U$. wightii (Wight) Lakshmin. and may be regarded as more dependable and useful criteria in studies on comparative morphology and taxomomy.

\section{REFERENCES}

Abdelgadir, H.A., Johnson, S.D. and Van Staden, J. (2012). Pollen viability, pollen germination and pollen tube growth in the biofuel seed crop Jatropha curcas (Euphorbiaceae). South African Journal of Botany, 79: 132-139. 

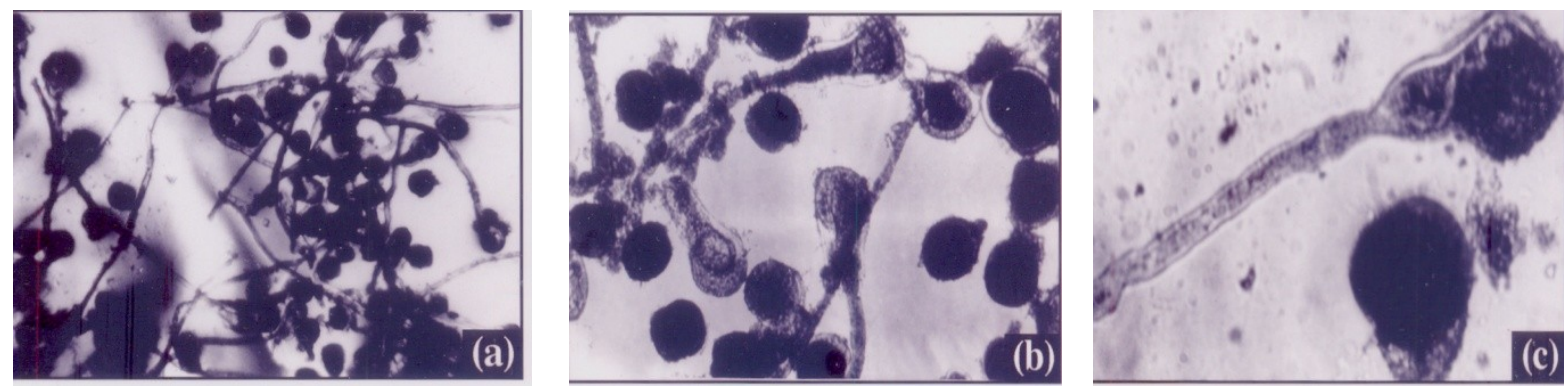

Fig 3. (a) - (b) Pollen germination. (c) Pollen tube formation of accession number 835 (Ramanagaram).
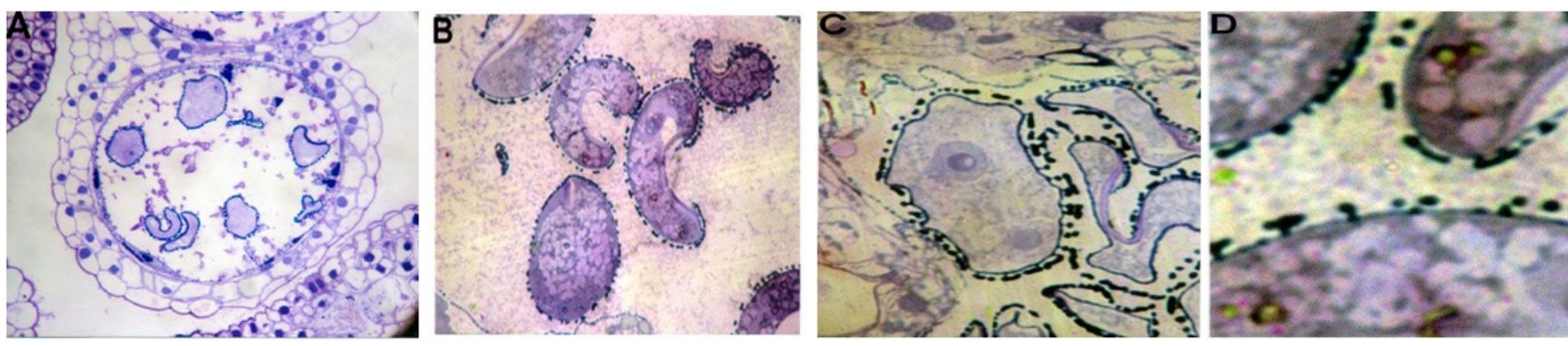

Fig. 4. Pollen development in Urginea indica accession number 835 (Ramanagaram). A - T.S. of Microsporangium. B - Development of Pollen grain. $\boldsymbol{C}$ - Pollen grain showing vegetative and generative nucleus. D - Pollen grains with tectum and collumellae.

Alcaraz, M.L.,;Montserrat, M, and. Hormaza, J.I.( 2011). In vitro pollen germination in avocado (Persea americana Mill.): Optimization of the method and effect of temperature. Scientia Horticulturae, Amsterdam, 130: $152-156$.

Airy- Shaw, H.K. (1965). Diagnoses of new families, new names etc. for the seventh edition of Willis's Dictionary, Kew, Bulletin, 18: 249 - 273

Beyhan, N. and Serdar, U. (2008). Assessment of pollen viability and germinability in some European chestnut genotypes (Castanea sativa L.). Hort Science 35: 171178.

Brewbaker J. L.and Kwack B. H. (1963). The essential role of calcium ion in pollen germination and pollen tube growth. American Journal of Botany 50: 859-865.

Carol.A.Furness and Paula. J. Rudall (2001). Pollen and other characters in monocot systematics. Grana, 40: 1$2,17-25$.

Ceter T., Ekici M., Pinar NM. and Ozbek F. (2013). Pollen morphology of Astragalus L. section Hololeuce Bunge (Fabaceae) in Turkey. Acta Botanica Gallica 160: 43 52.

Charles A.Knight., Rachel B. Clancy, Lars Gotzenberger, Leighton Dann, and Jeremy M.Beaulieu.(2010). On the Relationship between Pollen size and Genome size. Hindawi Publishing Corporation Journal of Botany 7 pages.

Fakhim, R.S.H.; Hajilou, J. and Zaare, N.F. (2011). Pollen germination and pistil performance in several Iranian peach cultivars. International Journal Agri Science 1: 71-77.

Harley, M. M., and M. S. Zavada. (2000). Pollen of the monocotyledons: selecting characters for cladistic analysis. 191-210.

Hauser, E. J. P., and Morrison, J. H. (1964). The cytochemical reduction of nitroblue tetrazolium as an index of pollen viability. American Journal of Botany, 51: 748-752.
Izzet Acar , Vijaya Gopal Kakani (2010). The effects of temperature on in vitro pollen germination and pollen tube growth of Pistacia spp. Scientia Horticulturae $125: 569-572$.

Kosenko, VN. (1999). Contributions to the pollen morphology and taxonomy of the Liliaceae. Grana 38: $20-30$.

Liang, S. Y. and Zhang, W. X. (1985). Pollen morphology of the genus Nomocharis and its delimitation with Lilium. Acta Phytotax. 23: 405 - 417.

Mehtap Tekşen, Zeki Aytac, Nur Munevver Pinar (2010). Pollen morphology of the genus Fritillaria L. (Liliaceae) inb Turkey Turk J Bot 34: 397- 416.

Moore, P.D., and Webb, J.A., (1978). An illustrated Guide to Pollen Analysis, Hodder and Stoughton, London

Muratovic E., Bogunic F., Soljan, D. Martin,J.,Vales,J.,Sonja Siljak Yakovlev (2010) Stomata and pollen grain characterstics of two endemic lilies:Lilium bosniacum and L.carniolicum (Liliaceae) Phytologia Balcanica 16: 285 $-292$.

Neetin Desai .,Hemant Kawalkar and Ghansham Dixit (2012)Biosystematics and evolutionary studies in Indian Drimia species Journal of Systematics and Evolution 50 (6): 512-518 .

Pupuleku B., Kapidani G., Kallajxhiu N., Naqellari P. and Turku S. (2010)_. Palynological Study of Pollen grains of Albania's Endemic plant Festucopsis serpentini (C.E.Hubbard) Melderis and Lilium martagon L. in three different habitats. Natura montenegrina 9: 431440.

Sayed-Mohammad Masoumi (2012) Exine Ultrastructure of Fritillaria (Liliaceae Juss) J. Basic. Appl. Sci. Res., 2 (2)1101-1109.

Seher Guven , Seda Okur, Mine Sezen Dem irel , Kamil Coskuncelebi , Serdar Makbul and Osman Beyazoglu (2014) Pollen morphology and anatomical features of Lilium (Liliaceae) taxa from Turkey Biologia 69/9: 1122-1133. 
Sevil Pehlivan, Hulya Özler (2003) Pollen morphology of some species of Muscari Miller (LiliaceaeHyacinthaceae) from Turkey Flora 198, 200-210.

Shiva Kameshwari M.N.,(2011). Pollen morphology in some members of Liliaceae. International Journal of Engineering Science and Technology. 3: 3825 - 3830.

Shiva Kameshwari, M. N. and Muniyamma, M. (2004). Karyomorphological Studies in Urginea indica Kunth. (Liliaceae) of Karnataka in India Beitrage Biologie der Pflanzen, 73: 377-394.

Sorkheh, K.; Shiran, B.; Rouhi, V.; Khodambashi, M.; Wolukau, J.N. and Ercisli, S. 2011. Response of in vi- tro pollen germination and pollen tube growth of almond (Prunus dulcis Mill.) to temperature, polyamines and polyamine synthesis inhibitor. Biochemical Systematics and Ecology 39: 749-757.

Troia A., Raimondo F.M., Castellano G. and Spadaro V. 2012. Morphological, karyological and and taxonomic remarks on Ferulago nodosa (L.) Boiss. (Apiaceae). Plant Biosyst. 146: 330 - 337.

Walker, J.W. and Doyle, J.A. (1975) The bases of angiosperm phylogeny: palynology. Annals of the Missouri Botanical Garden 62, 664-723. 